\title{
Correlation between polymer structures and functions of lignophenols
}

\author{
Keigo Mikame and Masamitsu Funaoka \\ SORST JST, Graduate School of Bioresources, Mie University, 1577, Kurima-machiya, Tsu, Mie 514-8507, Japan \\ Fax: +81-59-231-9517, E-mail: mikame@bio.mie-u.ac.jp
}

\begin{abstract}
A polymer structure and function of lignophenol was examined by various structural analysis of lignophenols fractionated with preparative SEC. The base unit of lignophenol is 1,1-bis (aryl) propane-2-O-aryl ether unit in all the molecular weight areas by FT-IR and NMR analysis. But the amounts of combined cresol and phenolic hydroxyl groups were increased with decreasing molecular weight of fractionated lignophenols.

The molecular morphology of lignophenol and conventional lignins were analyzed with SEC-MALLS system. The difference in the molecular weight of lignin determined by MALLS and conventional calibration methods using linear polystyrene standard can show the difference of molecular morphology of lignin. The molecular morphology of lignocresol was more spreading and linearity, compared with other lignins. It was suggested that this characteristic of lignophenol is concerned with thermoplasticity of lignophenol that other lignins do not have.
\end{abstract}

Key words: lignin, lignophenol, phase-separation system, molecular weight, molecular morphology

\section{INTRODUCTION}

Lignin is the most abundant natural polymer next to cellulose and exists in plant cell walls as one of the major constituents. It serves as cement between wood fibers, a stiffening agent within fibers, and a barrier to the enzymatic degradation of the cell wall. However, in contrast to the importance and potential of lignin in nature, lignin-based products have scarcely been in human life. This strange phenomenon is due to complicated structure and reactivity of lignin. Lignin is biosynthesized via random radical coupling of $p$-hydroxycinnamyl alcohols, which is initiated by enzymatic one-electron oxidation of phenolic hydroxyl groups. Thus lignin has a variety of inter-unit linkage and an amorphous three-dimensional network polymer. Furthermore, complicated modifications of the lignin structure are caused through isolation process from the cell wall. Presently kraft lignin which is the most abundant commercial lignin can be produced mainly as by-products in kraft pulping process. However the lignin from kraft pulping process are burned for the production of energy for pulping process, so they are not utilized as raw materials for chemicals. In order to utilize the kraft lignin as more valuable materials, the conversion of kraft lignin to functional polymers has been attempted by many researchers, ${ }^{1,2}$ but any industrial use of the lignin has not been accomplished.

Recently Lignin-based functional polymer (lignophenol) was derived from native lignins through the phase-separation process. ${ }^{3-6}$ This process includes a phase-separation reaction system composed of phenol derivatives and concentrated acid. In the process, native lignin was modified by selectively grafting phenol derivatives to benzyl position, the most reactive sites, to give lignophenol that remain the original interunit linkage of lignin and have high phenolic content. The lignophenol have several unique functions, which conventional lignins do not have, in spite of retention of the original interunit linkages, for example, solid-liquid transformation and high immobilization capability for proteins (enzyme). ${ }^{6}$ It is thought that the cause of these unique functions of lignophenol is attribute to molecular morphology of lignophenol. In this study, the correlation between these unique functions of lignophenols and polymer structures were investigated.

\section{EXPERIMENTAL}

2.1 Preparation of lignocresol

For solvation of lignin with phenol derivatives, 3 mol/C9 (phenyl propane unit of lignin) of $p$-cresol dissolved in acetone was added to defatted wood meal (spruce and birch) and acetone was evaporated with stirring. Sulfuric acid $(72 \%, 10$ $\mathrm{ml} / \mathrm{g}$ wood) was added to the mixture and the vigorous stirring was continued at $30^{\circ} \mathrm{C}$ for 60 min. The reaction mixture was rapidly poured to excess distilled water. The insoluble fraction was collected by centrifugation, washed with distilled water until neutral and lyophilized. The dried insoluble fraction was extracted with acetone. The acetone solution was then concentrated under reduced pressure and added dropwise to an excess amount of diethyl ether with stirring. The precipitated lignin derivative (lignocresol) was collected by centrifugation. Yields of lignocresol from spruce and birch were $27.5 \%$ of wood and $22.2 \%$ of wood respectively. 


\subsection{Fractionation of lignocresol}

Lignocresol was fractionated by preparative size exclusion chromatography (SEC) on Shimadzu LC-8A recycle preparative system equipped with Shimadzu SPD-10AUV/VIS detector. Shodex GPC KF-5002.5 (50 mm ID $x 300 \mathrm{~mm})$ was connected and tetrahydrofuran (THF) was used as an eluent [flow rate; $10 \mathrm{ml} / \mathrm{min}$ ]. The fractionated lignocresols solutions were evaporated to dryness under reduced pressure and dissolved in acetone. The acetone solutions were added dropwise to an excess amount of $n$-hexane with stirring. The precipitates were collected by centrifugation.

2.3 SEC-MALLS analysis

Size exclusion chromatogram of lignocresol was determined on a JASCO PU-1580 equipped with JASCO MD-1515 multi wavelength detector. Shodex GPC KF-801,802,803,804 (8 mm ID x 300 $\mathrm{mm}$ ) was connected in series and THF was used as an eluent [flow rate; $1.0 \mathrm{ml} / \mathrm{min}$ ].

MALLS analysis was performed on a DAWN-EOS multi-angle laser light scattering detector (Wyatt Technologies). The DAWN-EOS have 18 detectors, detector number $18,16,14,12$, $10,8,6,4$ and 2 were equipped with narrow band-pass interference filters to eliminate fluorescence. MALLS detector was combined SEC system. In addition, the SEC system was equipped with refractive index detector and photodiode array detector. Data acquisition and molecular weight calculation were performed using the ASTRA software (Wyatt Technologies). Molecular weight determination of lignins was calculated through the next equations.

$(\mathrm{RI})=(\mathrm{dn} / \mathrm{dc}) \times \mathrm{C} \times \mathrm{K}_{\mathrm{RI}}$

$(\mathrm{LS})=(\mathrm{dn} / \mathrm{dc})^{2} \times \mathrm{C} \times \mathrm{Mw} \times \mathrm{K}_{\mathrm{LS}}$

$\mathrm{Mw}=(\mathrm{LS}) /(\mathrm{RI}) \times 1 /(\mathrm{dn} / \mathrm{dc}) \times \mathrm{K}_{\mathrm{RI}} / \mathrm{K}_{\mathrm{LS}}$

LS: light scattering intensity

$\mathrm{dn} / \mathrm{dc}:$ refractive index increment

$\mathrm{C}$ : concentration of sample

KLS: calibration constant of MALLS apparatus

RI: intensity of refractive index detector

KRI: calibration constant of refractive index detector

In the MALLS analysis, $\mathrm{dn} / \mathrm{dc}$ is characteristic value of sample which has to be known from independent measurement. But $\mathrm{dn} / \mathrm{dc}$ is widely variable in the same lignin, because lignin is amorphous polymer. In this study, molecular weight was calculated by Mass method. In the Mass method, $\mathrm{dn} / \mathrm{dc}$ was calculated assuming that injected sample completely eluted on SEC analysis and the $\mathrm{dn} / \mathrm{dc}$ was assigned to the described above equations. All samples for SEC-MALLSS analysis were acetylated to prevent intermolecular association. In this study, SEC-MALLS system equipped with narrow band-pass interference filters to eliminate fluorescence was utilized for solving problems of optical anisotropy correction and influence of fluorescence. However, since it is difficult to determine absolute molecular weight of complicate lignins by even this SEC-MALLS system, the measured value from this system expressed as 'MALLS index'.

2.4 Contents of combined $p$-cresol and hydroxyl groups of fractionated lignocresols

${ }^{1} \mathrm{H}-\mathrm{NMR}$ analysis of fractionated lignocresols was performed on a JEOL JNM-A500 FT-NMR System. The amount of cresol induced was calculated based on the signal intensity of cresolic methyl protons (1.6-2.4 $\mathrm{ppm})$ against aromatic protons (7.8-8.4 ppm) of $p$-nitrobenzaldehyde (internal standard) on ${ }^{1} \mathrm{H}-\mathrm{NMR}$ spectra. ${ }^{5}$ The hydroxyl group contents were determined from phenolic acetoxyl proton signals $(2.0-2.4 \mathrm{ppm})$ and aliphatic acetoxyl proton signals (1.6-2.0 ppm) on ${ }^{1} \mathrm{H}-\mathrm{NMR}$ spectra of acetylated lignocresols. The cresolic methyl proton signals overlapped in the region of acetoxyl protons were estimated based on the relative intensity of cresolic methyl- to aromatic proton signals (6.3-7.8 ppm) in the spectra of original lignocresols.

2.5 FT-IR of spectra of fractionated lignocresols

FT-IR spectra of fractionated lignocresols were determined on Perkin Elmer Spectrum GX FT-IR Spectroscopy using $\mathrm{KBr}$ discs. The spectra were recorded from 400 to $4000 \mathrm{~cm}^{-1}$

2.6 Thermomechanical analysis (TMA) of fractionated lignocresols

Thermomechanical analyses of fractionated lignocresols were performed on a SEIKO EXSTAR $6000 \mathrm{TMA} / \mathrm{SS}$. About $5 \mathrm{mg}$ of a powdered sample was placed in an aluminum pan $(5 \mathrm{~mm} \varphi \times 2 \mathrm{~mm})$ and aluminum plate $(2 \mathrm{~mm} \times 2$ $\mathrm{mm}$ ) was put on the sample. The sample was compressed with the quartz probe with a $5 \mathrm{~g}$ load under a nitrogen stream and heating rate was carried on $2^{\circ} \mathrm{C} / \mathrm{min}$.

\section{RESULTS AND DISCUSSION}

\subsection{SEC-MALLS analysis of fractionated} lignophenols

Lignocresols from spruce and birch were fractionated into five fractions at second elution by recycle preparative SEC. These fractions and original lignocresols were analyzed by SEC-MALLS. The MALLS index of original lignocresol from spruce and birch were 31,900 and 17,200 , respectively (Table I, II), although weight average molecular weights of these lignocresols obtained on the same SEC system calculated with polystyrene standards were 12,900 and 8,200 , respectively. The proportions of MALLS index and weight average molecular weights calculated with polystyrene standards (MALLS / PS) of these lignocresols were 2.5 and 2.1 , respectively. It is known that same retention time in a SEC separation indicates the same hydrodynamic volume. Therefore, the same hydrodynamic volume with different molecular weight indicates a different density. Li et al. reported that molecular weights of phenolic resin determined by the SEC-MALLS analysis method were higher than that by polystyrene standard method. The difference in the molecular weight distribution determined by MALLS and 
conventional calibration methods can be explained by the phenolic compounds being more compact and dense than the polystyrene standard used. $^{8}$ Therefore spruce lignocresol was more compact and dense than birch lignocresol. Because hard wood lignin composed bifunctional syringyl units and trifunctional guaiacyl units has more linkages through $\beta$-ether bond than soft wood lignin composed only guaiacyl units, molecular morphology of hard wood lignin was linear than soft wood lignin.

The MALLS/PS values of fractionated lignocresols increased with increasing molecular weight (Table I, II). This results indicate that high molecular weight fraction of lignocresol is more compact and dense than low molecular weight fraction.

Table I Molecular weights of fractionated spruce ligncresols by SEC/MALLS method.

\begin{tabular}{|c|c|c|c|c|c|}
\hline & PS* Mw & MALLS index & MALLLS/PS & PS Mw/Mn & Yields \\
\hline $\mathrm{F}_{\mathrm{T}-1}$ & 66714 & 630350 & 9.45 & 2.57 & 10.5 \\
\hline $\mathrm{Fr}-2$ & 28513 & 88365 & 3.10 & 1.62 & 18.8 \\
\hline $\mathrm{Fr}_{\mathbf{r}} 3$ & 9592 & 22875 & 2.39 & 1.34 & 36.3 \\
\hline$F_{T}-4$ & 40,34 & 9474 & 2.35 & 1.20 & 25.2 \\
\hline Fr-5 & 2556 & 8224 & 3.21 & 1.44 & 9.1 \\
\hline original & $1 \quad 12922$ & 31940 & 2.47 & 3.08 & \\
\hline
\end{tabular}

Table II Molecular weights of fractionated birch ligncresois by SEC/MALLS method.

\begin{tabular}{lrrrrr}
\hline \multicolumn{7}{c}{ PS* } & Mw & MALLS index & MALLS/PS & PS Mw/Mn & Yields \\
\hline Fr-1 & 47253 & 203600 & 4.31 & 3.34 & 9.6 \\
Fr-2 & 13141 & 37045 & 2.82 & 1.42 & 24.9 \\
Fr-3 & 5536 & 10325 & 1.87 & 1.2 & 35.9 \\
Fr-4 & 2938 & 5549 & 1.89 & 1.14 & 19.1 \\
Fr-5 & 22.6 & 7193 & 3.16 & 1.53 & 10.5 \\
\hline original & 8196 & 17215 & 2.08 & 2.16 & \\
\hline
\end{tabular}

*polystylene standard method

Table III Hydroxyl groups and cresol contents of fractionated spruce lignocresols (mol/C9)

\begin{tabular}{lcccccc}
\hline & Fr-1 & Fr-2 & Fr-3 & Fr-4 & F -5 & Original \\
\hline Phenolic & 0.78 & 1.17 & 1.14 & 1.19 & 1.28 & 1.21 \\
Aliphatic & 1.00 & 1.08 & 1.09 & 1.09 & 0.98 & 1.13 \\
Total & 1.78 & 2.25 & 2.23 & 2.28 & 2.26 & 2.34 \\
Combined cresol & 0.46 & 0.60 & 0.71 & 0.73 & 0.78 & 0.70 \\
\hline
\end{tabular}

Table IV Hydroxyl groups and cresol contents of fractionated birch lignocresols (mol/C9)

\begin{tabular}{lcccccc}
\hline & Fr-1 & Fr-2 & Fr-3 & Fr-4 & Fr-5 & Original \\
\hline Phenolic & 1.09 & 1.14 & 1.25 & 1.44 & 1.51 & 1.47 \\
Aliphatic & 0.87 & 1.05 & 0.89 & 1.25 & 0.84 & 1.00 \\
Total & 1.96 & 2.19 & 2.05 & 2.69 & 2.35 & 2.47 \\
Combined cresol & 0.70 & 0.78 & 0.86 & 0.87 & 0.92 & 0.89 \\
\hline
\end{tabular}

3.2 Contents of combined cresol and hydroxyl groups of fractionated lignocresols

The contents of hydroxyl groups and $p$-cresol groups in the lignocresols molecular from spruce and birch are in Table III, IV respectively. The contents of hydroxyl groups and combined cresol in fractionated birch lignocresols were slightly higher than fractionated spruce lignocresols. The amounts of aliphatic hydroxyl group did not have a large difference between each fraction of fractionated lignocresols. However the amounts of combined $p$-cresol of high molecular weight fraction (Fr-1) were lower than low molecular weight fraction (Fr-5). The amounts of phenolic hydroxyl groups were also increased with decreasing molecular weight of fractionated lignocresols. The difference between the amount of total phenolic hydroxyl groups and the amount of combined $p$-cresol shows the amount of phenolic hydroxyl group of original lignin moiety. Therefore the amount of phenolic hydroxyl group originated from native lignin in low molecular weight fraction is more than that of high molecular weight fraction. In the phase separation system, phenol derivatives were grafted at benzyl position selectively following the cleavage of aryl ether bond or elimination of hydroxyl group at benzyl position. ${ }^{6}$ Thus it is thought that lignocresol of the low molecular weight fraction was derived from native lignin having many hydroxyl group or aryl ether bond, not carbon-carbon bond at benzyl position.

3.3 FT-IR spectra of fractionated lignocresols

As shown in Fig. 1, FT-IR spectra of original lignocresols and fractionated lignocresols from spruce had similar peak pattern, and the carbonyl absorption peak around $1720 \mathrm{~cm}^{-1}$ was not observed in the spectrum. However the carbonyl absorption peak was slightly appeared in birch original lignocresol, and the peak was strong in low molecular weight fraction $(\mathrm{Fr}-5)$. It was thought that this absorption was based on the carbonyl group and small amount of esther bond was contained in low molecular weight fraction. 3.4 TMA analysis of fractionated lignocresols

The TMA curves of lignocresols from spruce and birch revealed volume decreasing at $160^{\circ} \mathrm{C}-180^{\circ} \mathrm{C}$ due to transformation of solid state to clear liquid state (Fig. 2). ${ }^{7}$ On the other hand, spruce milled wood lignin (MWL), closely related to native lignin, cannot be transformed into fluid liquid under this condition. Because native lignin, three dimentional network polymer, are converted to linear-type polymers with high frequency of 1,1-bis (aryl) propane-2-O-aryl ethers during the phase separation treatment. The phase-transition point of low molecular weight fraction (Fr-5) of lignocresol from spruce and birch was lower temperature (spruce: $155^{\circ} \mathrm{C}$, birch: $145^{\circ} \mathrm{C}$ ) than main fraction (Fr-3) of fractionated lignocresols. The relationship between the phase-transition point and molecular weight was consist with the thermal behavior of fractionated kraft lignin, as investgated by DSC. ${ }^{9}$ Original lignocresol and high molecular weight fraction (Fr-1) had lower phase-transition point than $\mathrm{Fr}-3$, because original lignocresol and $\mathrm{Fr}-1$ has wider molecular weight distribution than other fraction (Table I, II), and low molecular weight moieties act as plasticizer. 

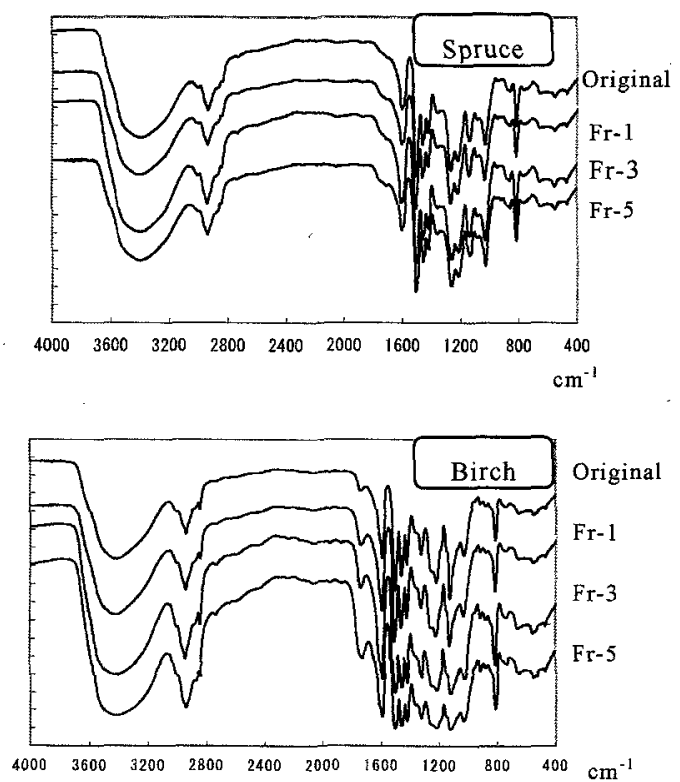

Fig. 1 FT-IR spectra of fractionated lignocresols.

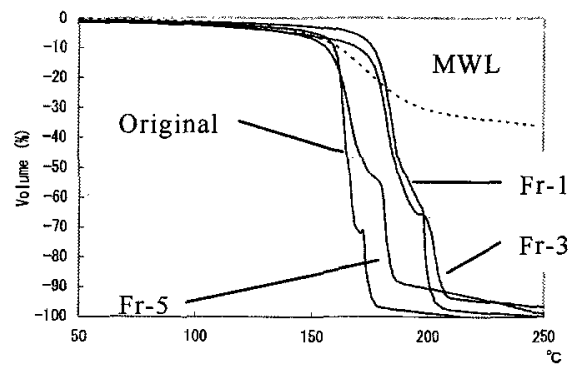

Fi.g. 2 TMA curves of fractionated spruce lignocresols.

3.5 SEC-MALLS analysis of industrial lignins and MWL

The MALLS index of kraft lignin was much higher than weight average molecular weights calculated with polystyrene standards. The MALLS index was 82,640, the MALLS/PS value was 11.3 (Table V). These values may be overestimate. But the effect of narrow band-pass interference filters is defined; the MALLS index by detector not equipped with narrow band-pass interference filters was 349,000 . This result indicates that the polymer structure of kraft lignin was more compact and dense than lignocresol. Because kraft lignin is derived through the cleavage of $C_{\beta}$-aryl ether bond and rearrangement by formation many carbon-carbon linkage under high energy condition, its polymer structure becomes compact, whereas structure of lignocresol is branched linear type structure based on $C_{\beta}$-aryl ether bond. Table $V$ is results of SEC-MALLS analysis of industrial lignins and MWL. The MALLS index of lignins derived under hard energy condition, for example explosion lignin and hydrotropic lignin, were higher than lignins derived under relatively mild condition, such as solvolysis lignin. The MALLS/PS value of explosion lignin and hydrotropic lignin were higher than solvolysis lignin. The MALLS/PS value of dioxane lignin low molecular weight fraction D1 was lower than high molecular weight fraction D2. These results indicate the lignins derived under high energy condition was converted to compact polymer structure because of cleavage of lignin interunit ether bond and rearrangement associated with formation of interunit carbon-carbon linkage.

The MALLS index and the MALLS/PS value of MWL are lower than industrial lignins. However MALLS/PS value of lignocresol were even lower than MWL, although it is said that MWL is most similar to native lignin. Therefore the molecular morphology of lignocresol was more spreading, compared with MWL. Because native lignin, three dimensional network polymer, are converted to branched linear type polymers with high frequency of 1,1-bis(aryl)propane-2-O-aryl ethers during the phase-separation process.

Table V Molecular weights of MWL and industrial lignins by SEC/MALLS method.

\begin{tabular}{|c|c|c|c|c|}
\hline & $\mathrm{PS}^{*} \mathrm{Mw}$ & MALLS index & MALLS/PS & PSMw/Mn \\
\hline Kraft lignin & 7358 & 82640 & 11.3 & 5.35 \\
\hline Solvolysis lignin & 7600 & 29535 & 3.89 & 2.52 \\
\hline Explosion Jignin & 9645 & 135850 & 14.21 & 6.08 \\
\hline Dioxane lignin Dl & 7824 & 37775 & 4.83 & 3.52 \\
\hline Dioxane lignin D2 & 10387 & 110400 & 10.63 & 5.05 \\
\hline Hydrotropic lignin & a 6414 & 128900 & 20.09 & 3.41 \\
\hline MWL spruce & 4632 & 19985 & 4.32 & 1.81 \\
\hline MWL birch & 6925 & 30020 & 4.34 & 2.18 \\
\hline
\end{tabular}

*polystylene standard method

\section{References}

[1] H. Nimz, 'Wood Addhes. Chem. Technol.', A. Pizzi Ed., Ch.5, Dekker, New York, 245 (1983).

[2] P.C. Muller, S.S. Kelly and W.G. Glasser, J.Adhes., 17, 185-206(1984)

[3] M. Funaoka and I. Abe, Tappi Journal 72, 145-149(1989).

[4] M. Funaoka, M. Matsubara, N. Seki and S. Fukatsu, Biotechnol. Bioeng., 46, 545-552 (1995). [5] M. Funaoka and S. Fukatsu, Holzforschung 50, 245-252 (1996).

[6] M. Funaoka, Polymer International, 47, 277-290 (1998).

[7] Y. Nagamatsu and M. Funaoka, Sen'I Gakkaishi, 57, 75-81 (2001)

[8] P.Li, D. W. Coleman, K. M. Spaulding, W. H. McClennen, P. R. Stafford and D. J. Fife, $J$. Chromatography A, 914, 147-159 (2001).

[9] H. Hatakeyama, K. Iwashita, G. Meshitsuka and J. Nakano, Mokuzai Gakkaishi, 22, 618-624 (1975)

(Received February 16. 2007;Accepted July 12, 2007) 